\title{
Nuevas perspectivas metodológicas para el estudio del habla egocéntrica
}

\author{
JUAN DANIEL RAMÍREZ \\ Universidad de Sevilla
}

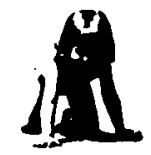

\section{Resumen}

Las hipótesis de Vygotski sobre el papel del habla egocéntrica en la regulación de las acciones representa uno de los temas de mayor interés para los estudiosos del binomio pensamiento-lenguaje. Para abrir nuevas vías al análisis de este campo del lenguaje es necesario reflexionar sobre una serie de cuestiones fundamentales, sin las cuales el sentido de estas hipótesis se desvirtúa. Estas quedan reflejadas en los siguientes puntos: a) definición vygotskiana de babla egocéntrica (indiferenciación entre el habla para los demás y el habla para uno mismo ); $b$ ) requisitos conversacionales elicitadores de este tipo de babla, los cuales evidencian su origen social (i. e., incremento del número de emisiones egocéntircas cuando el niño actúa en compañía de otros niños); y por último, c) el papel del contexto extralingüístico (i. e., objetos físicos sobre los que se actúa). Dada la gran dependencia del discurso infantil de la situación concreta, el contexto extralingüistico es analizado como un requisito conversacional más, responsable del mantenimiento de la conversación (babla social) y del babla egocéntrica asociada a ésta. Finalmente, la revisión de estas cuestiones invita a desarrollar una perspectiva dialógica de análisis a partir de las nociones de diálogo, habla interna y emisión de Bajtin. La perspectiva dialógica permitirá analizar las emisiones de carácter egocéntrico en el contexto lingüistico y/o conversacional en que emergen.

Palabras clave: Perspectiva dialógica, Habla egocéntrica, Habla privada, Requisitos conversacionales, Contexto extralingüistico.

\section{New methodological approaches in the study of egocentric speech}

\section{Abstract}

$V y g o t s k i$ 's hypotheses on the role of egocentric speech $(E \dot{S})$ in regulating actions represent some of the most interesting topics in the study of the thought-language relationship. In order to open up new possibilities to the analysis of ES, it is necessary to consider some important issues so that these hypotheses are not misunderstood. These refer to the following points: a) Vygotski's definition of $E S$ (indiferentiation between speech directed to others and speech to oneself); b) the role of conversational requirements in the production of egocentric utterances (i.e., the number of $E S$ utterances increases when children acts together); $c$ ) the role of extralinguistic context (i.e., events and objects in the physical enviroment). Since child discourse closely dependent on concrete situations, extralinguistic context is analised as one more conversational requirement necessary to maintain a conversation (social speech) and for $E S$, its more direct associate. Finally, the review of these issues allow us to propose a new perspective of study from Bakhtin's dialogical perspective approach to the study of language. It allows to analyse egocentric utterances in relationship with the linguistic and conversational context in which they are performed.

Keywords: Dialogical perspective, Egocentric speech, Private speech, Conversational requirements, Extralinguistic context.

Dirección del autor: Laboratorio de Actividad Humana. Universidad de Sevilla. Facultad de Psicología. Avda. San Francisco Javier, s/n. 41005 Sevilla. 


\section{INTRODUCCION}

Los recientes avances en psicolingüística evolutiva invitan a reconsiderar ciertos aspectos del habla infantil que en el pasado recibieron una especial atención y que, sin embargo, han sido desestimados durante muchos años por los psicólogos y estudiosos del binomio pensamiento-leguaje. El habla egocéntrica (HE) y su influencia en el desarrollo de la función de autorregulación es uno de estos temas que el psicolingüísta debe abordar en profundidad, si desea explicar la compleja relación existente entre ambos términos del binomio. Las hipótesis de Vygotski (1932, en prensa) acerca de esta interesante cuestión componen otro hecho del pasado que debe ser igualmente reconsiderado en el momento actual.

Para abordar el estudio del HE es recomendable iniciar un acercamiento al mismo desde la revisión de algunos aspectos metodológicos frecuentemente olvidados por los investigadores occidentales que han estudiado este problema. Esta revisión significa, en muchos sentidos, una vuelta a su origen, tal y como fue planteado por el propio Vygotski. Para alcanzar este objetivo, es necesario abordar igualmente una serie de cuestiones relacionadas con el concepto de HE, con su papel en el proceso de autorregulación y con su origen social. A mi juicio, todas ellas necesitan de una metodología nueva, que no desdibuje la riqueza de las hipótesis vygotskianas y que eleve a un primer plano el enfoque sociogenético que las sustenta. En el presente artículo me propongo revisar una serie de aspectos, que considero necesarios para el desarrollo de dicha metodología. Estos pueden resumirse en los siguientes puntos: una aproximación al concepto de HE desde una perspectiva social y funcional; una breve descripción de los requisitos conversacionales (o dialógicos) que son necesarios para observar la producción de emisiones egocéntricas ${ }^{1}$; el análisis del papel que cumple el contexto extralingüístico en la producción de dichas emisiones; $y$, por último, una aproximación al estudio del diálogo como factor desencadenante del HE. Para abordar este último punto expondré algunas de las aportaciones del filósofo y semiólogo Mijail Bajtin (Volosinov/Bajtin, 1986) acerca de un tema que preocupó por igual a ambos autores, el babla interna. Las ideas de Bajtin, a propósito del habla interna, se encuentran tan estrechamente relacionadas a las del propio Vygotski que, como James V. Wertsch apunta en esta misma monografía, pueden ser empleadas por los psicólogos estudiosos del enfoque sociocultural como extensiones de algunos de los puntos de vista de Vygotski. Su perspectiva dialógica dará que hablar a los estudiosos del lenguaje en los próximos años y promete abrir un interesante debate que ya deja sentir su influencia en diversos ámbitos de las ciencias sociales.

\section{ACERCA DE LA DEFINICION DE HABLA EGOCENTRICA}

En las dos últimas décadas, el término habla privada ha estado de moda entre los estudiosos del desarrollo del proceso de autorregulación, en lugar del más clásico de habla egocéntrica (HE), extensamente popularizado por Jean Piaget en la década de los años veinte. Los psicólogos soviéticos siguieron utilizando este último, si bien con un significado distinto, a partir 
de la obra de Lev S. Vygotski, Pensamiento y lenguaje, publicada, como es bien sabido, poco después de su muerte en 1934. El uso del nuevo concepto (habla privada) ha alcanzado tanta difusión entre los psicólogos occidentales que hoy somos pocos los defensores de la noción más clásica de HE. Expondré brevemente las razones por las que me inclino a defender el término tradicional y a recomendar prudencia en el uso del más reciente y ampliamente difundido en nuestra comunidad científica.

La noción de habla privada fue introducida por Flavell en los años sesenta (Flavell, 1966), con el objeto de marcar el carácter funcional de un tipo de lenguaje orientado exclusivamente al control y regulación de la tarea. El problema contaba con importantes antecedentes hoy olvidados. Reese (1962), por ejemplo, se había interesado por el uso de ciertas palabras que parecían funcionar como elementos mediadores de las acciones implicadas en la resolución de problemàs. A través de los escritos de Reese y de Flavell y con un nuevo término acuñado, estas formas idiosincráticas de habla tendían a verse, exclusivamente, desde una perspectiva mediacionista primero y cognitivista, más tarde. Con el interés que la psicología cognitiva suscitó a partir de aquellos años, los estudiosos del tema analizaron el HP desde una perspectiva procesual, sin considerar para nada la dimensión sociogenética que proponía Vygotski. El habla privada interesaba en tanto que en ella se aglutinaban un conjunto de emisiones directamente suscitadas por la propia tarea. Yo diría que una gran parte de los estudios revisados por Fuson (1979) e incluso muchos de los trabajos realizados posteriormente, se han centrado en la dimensión mediacionista ${ }^{2}$ y/o cognitiva de esta forma de habla, en menoscabo de sus componentes sociales. En este contexto, los signos y emisiones producidos en situación de resolución de problemas se han analizado considerando únicamente su eficacia resolutiva, sin tomar en consideración sus propiedades dialogales. Esta posición estrictamente cognitivista, unida a la orientación nominalista que el adjetivo "privado" impone, ha llevado a primar lo individual, funcional e intrapsicológico sobre otros factores asociados a su origen social. En mi opinión, todos estos aspectos han influido de forma más o menos inconsciente sobre los investigadores, muchos de los cuales imprimieron una orientación solipsista a unas hipótesis que, como las de Vygotski, no pueden ser abordadas más que desde una perspectiva sociogenética.

Desde mi óptica personal creo interesante seguir usando el término HE, a la vez que conviene limitar el de $\mathrm{HP}$ - si es que se desea seguir empleando- a unos ámbitos más restringidos de la producción verbal del niño, tales como los susurros (whispers and mutterings) y formas próximas a la subvocalización. En ellas se puede observar una clara diferenciación entre el habla para los demás y el habla para si, así como el correspondiente proceso de interiorización de esta última.

Más de un investigador en el tema podría pensar que esta disposición al uso del término clásico, representa una opción conservadora que se verbalizaría de la siguiente manera: mientras las cosas no estén algo más claras, no conviene introducir términos nuevos que hagan más farragoso el acercamiento al problema. Sin embargo, la cuestión que aquí trato de plantear se relaciona directamente con el grado de contacto social entre hablantes, en el momento en que el investigador registra producciones catalogadas como HE. Muchas de las producciones verbales emitidas por el niño 
con fines reguladores se efectúan en el marco de la tarea que éste realiza, sin dirigirse a una audiencia específica ni buscar el contacto visual con el compañero más cercano. Además, estas mismas emisiones presentan un marcado contenido privado, pues lo que se dice está en estrecha conexión con lo que en ese momento se hace. No obstante, hay algo en todas ellas que llama poderosamente la atención: se profieren con la suficiente intensidad de voz como para que otro u otros pueda oirlas. Esta forma de habla deja en el observador la difusa impresión de que el hablante supone la existencia de un oyente hipotético implicado, de alguna forma, en lo que el primero dice acerca de la propia tarea. La ilusión de ser comprendido, como se denomina a la actitud que subyace al HE, nos pone en la pista de un tipo de emisión conectada genéticamente con el habla social (HS). El hecho de que estas emisiones comporten contenidos eminentemente privados $y$, por ende, estrechamente relacionados con la acción en curso, no niegan, sin embargo, una cierta proyección pública. De ahí que esa indiferenciación entre el habla para los demás y el habla para uno mismo sigue siendo un rasgo de interés que Vygotski mejor que nadie supo ver en el $\mathrm{HE}$.

\section{Requisitos conversacionales para la producción de habla egocéntrica}

Para conseguir un marco adecuado en el que observar la producción de $\mathrm{HE}$ es importante volver a retomar los orígenes de estas investigaciones y centrarse en las condiciones que Vygotski consideró fundamentales para su observación. Como es sabido, sus experimentos cruciales tuvieron como objetivo demostrar la raíz social del babla interna, rompiendo tanto con el mecanicismo de Watson a propósito de este mismo tema, como con el reduccionismo cognitivista de Piaget. Sus trabajos, así como los de los investigadores occidentales que han tratado de contrastar sus hipótesis (ver la revisión de Fuson, 1979), ponen de manifiesto que son necesarias ciertas condiciones para el diálogo, si se desea observar la estructura y función del HE. La mayor parte de estas condiciones, a las que denominaré requisitos conversacionales, como ya he expresado en otra ocasión (Ramírez, en prensa), se extraen directamente de los experimentos cruciales del propio Vygotski. Así sucede al menos con los tres primeros requisitos conversacionales; el cuarto se ha inferido de algunas investigaciones actuales (Wertsch, 1979; Wertsch, 1980; Wertsch y Stone, 1985; Goudena, 1983) y el quinto y último, ha sido estudiado por el autor de este artículo y se encuentra aún en fase de investigación teórica y experimental. En orden a su novedad y al posible debate que pueda suscitar, este último será analizado posteriormente. Se exponen a continuación los cuatro requisitos, ordenados de menor a mayor grado de complejidad y posible dependencia jerárquica:

1. La posibilidad de interacción social. Como algunos estudios han venido a demostrar (Kohlberg, Yaeger y Hjertholm, 1968; Fuson, 1979; Berk y Garvin, 1984; Frauenglass y Díaz, 1985; Díaz y Padilla, 1985), el número de producciones de $\mathrm{HE}$ se incrementa o disminuye en función de las posibilidades de interacción social que la situación ofrece. Un niño que trabaja en solitario (i. e., resolución de problemas) en un aula de su parvulario se verá menos estimulado a hablar que otro rodeado por los compañeros con los que se relaciona cotidianamente. 
2. Requisitos ambientales. Cuando las condiciones físicas del ambiente dificultan la comunicación, se obstaculiza indirectamente la producción de HE. Vygotski demostró que los sonidos de cierta intensidad, aún en el caso de que éstos fueran armónicos (música interpretada por una banda, como sucede en su experimento), además de dificultar que los niños pudieran oirse entre sí, inhibía la producción de habla egocéntrica.

3. El diálogo. Si el niño percibe que no es comprendido por su audiencia, el número de emisiones HE descenderá bruscamente. Como Vygotski también pudo comprobar, cuando el niño que estaba siendo observado se encontraba rodeado por otros niños con los que no podía comunicarse (i. e., sordomudos, hablantes de una lengua extranjera), el coeficiente de HE descendía hasta un nivel cercano a cero.

4. El otro como colaborador potencial. Este requisito ha sido analizado por los estudiosos de la zona de desarrollo próximo. Wertsch y cols. (Wertsch, 1979; Wertsch y Hickmann, 1987; Wertsch y Stone, 1985) han observado emisiones de $\mathrm{HE}$ en sujetos de corta edad (tres años aproximadamente) que resuelven problemas con la ayuda de un adulto. A lo largo del tiempo que duraba la tarea se podían observar dos fases perfectamente diferenciables: en una primera fase, el niño era guiado por el adulto (generalmente su madre) mediante orientaciones y sugerencias que invitaban a explorar el modelo de un puzzle, la relación entre sus diferentes piezas, etc. (beterorregulación). Después de un período de transición, el pequeño asumía las funciones de guía y orientación de la propia tarea, llegando a producir emisiones dirigidas a sí mismo, marcadamente autorreguladoras y, por tanto, de características similares a las emitidas por la madre al inicio de la colaboración. Goudena $(1983,1987)$, en estrecho paralelismo con estos hallazgos, pudo comprobar que la tendencia a hablarse a sí mismo aumentaba cuando el niño (cuatro años de edad) percibía al experimentador como un colaborador potencial. Su disposición a ayudar ante las dificultades que pudieran surgir, se convertía indirectamente en una invitación al monólogo en el que predominaban las emisiones orientadas a la regulación de la tarea.

Todo hace pensar que cada uno de estos requisitos representa un nivel de complejidad mayor y, por tanto, incluye en sí las caracteristicas del anterior. En primer lugar es fundamental que el niño no esté solo ante el problema a resolver, si queremos que elicite algún tipo de verbalización egocéntrica (posibilidad de interacción social). Además, las condiciones del entorno deben ser las adecuadas para que físicamente sea posible la comunicación (requisitos ambientales). Una vez que las condiciones físicas son las adecuadas, es necesario que haya uno o varios interlocutores para iniciar un posible diálogo (diálogo como requisito). A su vez, el diálogo representa una apertura hacia posibles formas de cooperación. En base a este nuevo requisito, el «otro" (el destinatario, la audiencia, etc.) se percibe por el niño como un colaborador hipotético, presto a actuar de apoyo y guía en el desarrollo de las acciones.

\section{El contexto extralingüistico como requisito conversacional}

Un aspecto que a mi juicio ha sido poco considerado por los estudiosos del HE es el contexto extralingüístico en el que este tipo de emisiones emer- 
ge. El elevado grado de dependencia contextual del habla infantil está siendo muy considerado en la actualidad para analizar el desarrollo de las destrezas comunicativas. Este aspecto es esencialmente relevante cuando se trata de explicar el desarrollo del discurso infantil (Karmiloff-Smith, 1979; Hickmann, 1980, 1980, 1985), la descontextualización de los procedimientos semióticos de mediación, así como su papel en la transformación de los procesos cognitivos superiores (Wertsch 1985, 1985; en prensa).

Como ya es sabido, la mayor parte de las producciones de $\mathrm{HE}$ nacen en estrecha conexión con la tarea y con los objetos que se manipulan en ella (puzzles, estímulos a clasificar, etc.). Estos últimos representan una parte importante de la situación inmediata en la que el niño se desenvuelve. La dependencia contextual de que hace gala el habla infantil y la función de regulación que el $\mathrm{HE}$ cumple en el curso de las acciones invitan a analizar el contexto físico como un requisito metodológico a considerar. El siguiente ejemplo puede darnos algunas claves al respecto: dos niños están trabajando en tareas iguales o muy similares. Entre ellos comienza un diálogo acerca de lo que cada uno hace. Dada la similitud entre sus respectivas tareas y la falta de habilidad para mantener la conversación fuera de toda referencia a los elementos de las mísmas, desde el comienzo de la conversación emergen formas deícticas («éste de aquí»; «aquello», "estas — piezas- son mías", etc.) que en la mayor parte de los casos estarán acompañadas de gestos extralingüísticamente orientados (i. e., señales con el dedo índice hacia determinadas piezas del puzzle). Los sucesos y objetos que componen el contexto son, pues, fundamentales para el inicio y desarrollo de la conversación. Cada emisión de HE que observemos estará en conexión con el diálogo que los niños mantienen y, por tanto, con los objetos de los que se habla. Este razonamiento conduce directamente a una segunda pregunta acerca de la evolución que tomará el habla social y su asociado, el $\mathrm{HE}$, cuando estando presentes todas las condiciones para el diálogo, no se tenga un acceso visual directo a sus respectivas tareas. Pongamos a dos niños a actuar juntos manteniendo todos los requisitos antes descritos: no hay ruidos ambientales que distorsionen la comunicación; ambos son compañeros que conviven juntos en la clase; se observa, además, una cierta disposición a colaborar entre ellos, etc. No obstante, a pesar de que se dan todas estas condicones, mediante la adecuada manipulación experimental dificultamos la visión directa de sus respectivas tareas. $\mathrm{Si}$, como es sabido, los niños tienen dificultades para superar las limitaciones de su contexto extralingüístico, es de esperar que el diálogo descienda y consiguientemente se reduzca la posibilidad de una emisión fluida de HE.

Expondré brevemente un estudio en el que se tomó como variable independiente el contexto físico en que tiene lugar el desarrollo de una determinada tarea (dibujo) y como variable dependiente las distintas producciones verbales, tanto sociales como egocéntricas, presentes en el curso de su realización (Ramírez, en prensa).

Se seleccionaron veinticuatro niños hispanos monolingües en edades comprendidas entre 4;5 y 5 años de edad que asistían a un kindergarten incluido en el programa Head Start en Solana Beach (Southern California). La tarea consistía en realizar un dibujo de sus respectivas familias. Los niños trabajaban en parejas, sentados en una mesa circular situada en una esquina del aula en que pa- 
saban la mayor parte del día. Para que pudieran trabajar con comodidad, se acondicionó un espacio con unos cuantos muebles de baja altura (estanterías) que si bien creaba condiciones para concretarse en la actividad, en ningún caso los aislaba de su entorno cotidiano. Bastaba con ponerse de pie para ver al resto de sus compañeros de clase. Después de mantener una conversación con cada niño acerca de los distintos mienbros que componían su familia (padre, madre, hermanitos, abuelo, etc.), se les invitaba a que los dibujaran. Los 24 sujetos observados fueron distribuidos en dos grupos (control y experimental), con doce parejas en cada uno. A todos ellos se les dijo que podía hablar si querían, que unos niños preferian hacerlo porque asi dibujaban mejor, mientras que otros preferían no hablar ${ }^{3}$. En el grupo experimental se introdujo una modificación consistente en obstaculizar la visión que cada niño tenía de la tarea del otro. Entre ambos se interponía una pieza rectangular de cartón $(100 \mathrm{~cm}$ de largo $\times 25 \mathrm{~cm}$ de alto aproximadamente) en posición vertical. Su altura era tal que permitía ver el cuello y la cabeza del compañero, pero impedía una visión directa de su dibujo. Igual se hizo con los sujetos del grupo de control, invitándoles a éstos también a que hablaran cuanto quisieran, si ese era su deseo. Se les demostraba que no tenían dificultad para hablar, pidiéndoles que se miraran el uno al otro. Igualmente, el experimentador les decía que podían mirar cuanto quisieran el dibujo del compañero o, por el contrario, enseñarles su propio dibujo. Con la inclusión de este obstáculo se trataba de manipular las condiciones contextuales de la tarea. El diseño cumplía todos los requisitos que permiten establecer una comunicación fluida entre los mienbros de la pareja. En el caso del grupo experimental, la pared de cartón respetaba los cuatro requisitos descritos, pero interfería un quinto: la visión directa y sin interrupciones de las respectivas tareas (qué personaje se realizaba en cada momento, la calidad de sus trazos, etc.). $\mathrm{He}$ de insistir en que a los niños del grupo experimental no se les impedía la posibilidad de ver el dibujo de su compañero; simplemente se le creaba una cierta dificultad. Cada miembro de la pareja podía incorporarse y mirar por encima del muro o, por el contrario, alzar su papel y enseñar su trabajo como de hecho sucedió en varias ocasiones. Durante la fase de instrucción a los niños se les invitó igualmente a mirar por encima de la pieza de cartón, en el caso en el que desearan mirar el dibujo del contrario.

La falta de visión directa del dibujo del otro, bloqueaba la posibilidad de tener un conocimiento rápido y continuo del contexto del que se habla, impidiendo disponer de un tema de conversación. De suyo, los niños del grupo de control hablaron significativamente más que los del grupo experimental. En medio de una conversación sobre los personajes del dibujo, era frecuente observar, entre los sujetos del primer grupo, una producción verbal de marcado cariz egocéntrico, cuyo contenido estaba claramente relacionado con la tarea en curso. Frases como las que se presentan a continuación, son un buen ejemplo de expresiones que se efectuaban mientras el niño dibujaba, sin que se observara intención explícita de dirigirse al compañero (i. e., no se observaba contacto visual cara a cara entre ambos):

1. "Me salió mal mi mamá."

2. "Yo voy a hacer a mi papá."

3. "Uhm, ...su manita."

(El niño se percata de haber olvidado dibujar la mano de la figura sobre la que trabaja.)

Las razones por las que los niños del grupo de control hablaron significativamente más (habla social + habla egocéntrica) parecían estar en la ac- 
cesibilidad que tenían al dibujo del compañero. Este configuraba el contexto físico y era, a su vez, el motivo del diálogo. Cuando los niños se encontraban inmersos en un tema de conversación, jalonaban constantemente sus preguntas, respuestas y afirmaciones con deícticos y gestos extralingüísticamente orientados. Los niños decían frases tales como:

4. «Este es mi papá.»

5. «¿Qué es eso?"

6. «iMira!, ¡mira!»

(Exclamaba mientras señalaba con su dedo índice el dibujo de su compañero.)

Los niños del grupo de control podían conversar más fácilmente entre ellos, porque disponían extralingüísticamente de los temas de conversación. El soporte del diálogo estaba en las figuras reflejadas en su papel o el del compañero. En estas circunstancias, iniciar una conversación resultaba bastante fácil; bastaba señalar con el dedo o con el extremo del lápiz la figura sobre la que se conversaba. En el caso del grupo experimental, esta referencia al contexto se veía dificultada por la existencia de la pared de cartón que les obligaba a incorporarse para ver el dibujo del otro o enseñar el suyo, levantando el papel por encima de la misma. En esas condicones, era inevitable que la conversación (habla social) terminara decayendo y, con ella, su más directo asociado, el habla egocéntrica.

\section{ESTRUCTURA CONVERSACIONAL DEL HABLA EGOCENTRICA}

En los apartados anteriores he descrito los cinco requisitos que en mi opinión deben ser tomados en consideración cuando se pretenden observar emisiones de HE. Sin embargo, una vez obtenida una cierta producción discursiva por parte del niño, surge otro problema referido a la forma en que hemos de proceder para su análisis. Como veremos a continuación, existen dos perspectivas metodológicas para abordar este tipo de estudios, a las que denominaré monológica y dialógica, respectivamente. Tales denominaciones se inspiran directamente en las nociones de diálogo y habla interna propuestas por el también soviético Mijail Bajtin (Volosinov/Bajtin, 1986). Ambas perspectivas abordan como unidad de estudio la emisión (utterance), si bien existe una oposición marcada entre ellas que se puede describir en los siguientes términos:

mientras que la orientación monológica toma como punto de referencia el estudio de la emisión aisladamente, la dialógica considera igualmente las relaciones entre emisiones en el contexto del diálogo.

La perspectiva monológica opera sobre producciones aisladas, considerándolas por el sentido ${ }^{4}$ que cada una de ellas tiene en el curso de la acción. Desde esta perspectiva, lo que se pretende, sobre todo, es analizar la función que cumple el contenido semántico de la emisión en el desarrollo de la tarea (función de planificación de la acción en curso, de descripción alguna de sus faceta, de evaluación, etc.). En algunos de los ejemplos to- 
mados de la investigación comentada en el apartado anterior (Ramírez, en prensa) se observaron oraciones planificadoras que marcaban la orientación de la acción inmediata (yo voy a hacer a mi papá), o, como en otros casos, oraciones que evaluaban la parte de la tarea realizada (me salió mal mi papá). Dado el enorme interés que ha existido en psicología por relacionar lenguaje y acción, es natural que esta perspectiva haya alcanzado un mayor desarrollo e igualmente un alto grado de popularidad entre los estudiosos del tema. Se podría añadir que el tratamiento monológico de la producción verbal del niño no ha variado sustancialmente desde las primeras observaciones realizadas por Piaget, aparecidas en Le langage et la pensée chez l'enfant (1924). La originalidad del planteamiento. vygotskiano y las críticas a Piaget fueron, sobre todo, más de orden teórico que metodológico. Se puede decir que Vygotski continuó aislando producciones egocéntricas en una forma muy similar a como lo hacía el primero. El número total de estas producciones se transformaba en un coeficiente que permitía conocer la relación existente entre ambas formas de habla (HS y HE). Esta metodología, que no ha sufrido grandes cambios desde sus orígenes, consiste sustancialmente en clasificar las emisiones en sociales y egocéntricas, considerando a priori que estas últimas cumplen funciones reguladoras o llegarán a adquirir dichas funciones, en el proceso de diferenciación de ambas formas de habla: A partir de los estudios de Kohlberg y cols. (1968), los investigadores se esforzaron por conseguir una clasificación más funcional del HE, atendiendo al papel que cada emisión cumple en el desarrollo de la acción. Como ya he puntualizado, son formas de análisis centradas en la estructura semántica interna de cada emisión: si ésta es o no relevante para la tarea, si simplemente describe la actividad propia o es planificadora de la acción en curso, si enuncia rasgos distintivos de la misma, etc. (Díaz y Padilla, 1985; Frauenglass y Díaz, 1985; Fuson, 1979; Goudena, 1983; Kohlberg, Yaeger y Hjertholm, 1968; Ramírez, en prensa; Vygotski, 1983).

Se podría resumir esta perspectiva diciendo de ella que se centran básicamente en el análisis de cada acción en particular, atendiendo tanto a las emisiones que en ella se profieren como el conjunto de operaciones la hacen posible ${ }^{5}$. Parafraseando a Habermas (Habermas, 1987) añadiré que el objeto de conocimiento que el investigador aborda es el habla circunscrita al marco de las acciones teleológicas, de las acciones dirigidas a fines. Con demasiada frecuencia se olvida que la función originaria del habla es la comunicación y su presencia en este tipo de acciones se debe fundamentalmente a que, en muchas ocasiones, las acciones teleológicas se producen en marcos comunicativos. Lo que más ha interesado a los investigadores ha sido conocer el grado de eficacia del habla con contenido privado dentro del complejo de las acciones, sin considerar las interacciones sociales que la han precedido. Se da la extraña paradoja de defender el origen social del HE sin prestar demasiado interés por el conjunto del diálogo en que la producción egocéntrica emergen. De cada emisión registrada, el observador distingue, en primer lugar, si se dirige explícitamente al oyente, a la audiencia (i. e., contacto cara a cara entre interlocutores, referencia verbal al oyente) $o$, más bien, parece dirigirse al emisor mismo. Se determina, de este modo, si la emisión pertenece a una de las dos categorías básicas (HS o $\mathrm{HE}$ ). A continuación el investigador procede a analizar su contenido $\mathrm{y}, \mathrm{a}$ través de él, conocer la función que, como ya apunté anteriormente, la emi- 


\section{8}

sión cumple en el marco de la acción en curso (planificación, evaluación, descripción, etc.). En un esfuerzo por depurar la metodología, algunos investigadores han recurrido a teorías que tienen un considerable peso específico dentro del panorama actual de las ciencias del lenguaje, como es el caso de la teoría de los actos del habla (Pellegrini, 1980; Pellegrini y Destefano, 1979).

La pregunta que a estas alturas de la investigación sobre el tema cabe hacerse es si existe alguna alternativa a la perspectiva monológica hasta ahora descrita. Mi respuesta, en este sentido, es afirmativa. La alternativa a una forma de análisis centrada en la emisión (perspectiva monológica) estaría en un enfoque complementario que considerase por igual a ésta y al diálogo en que se genera. Un enfoque así no cambiaría la unidad de estudio, sino que prestaría una especial atención al contexto conversacional en que los enunciados se originan. Como ya he apuntado anteriormente, algunas de las propuestas de Bajtin resultan muy sugerentes y atractivas para los estudiosos del HE por muy diversas razones; cabe resaltar entre otras, el enorme paralelismo existente entre su perspectiva dialógica y las hipótesis del propio Vygotski. Entre ambos autores se dan una serie de coincidencias que van más allá del tiempo, el lugar y otros condicionamientos históricos en que desarrollaron sus trabajos. Al igual que Vygotski, Bajtin ha sido conocido muy tardíamente en occidente, y sus trabajos considerados de gran interés por un número considerable de estudiosos de las ciencias sociales (Holquist, 1981; Wertsch, 1985, 1985, en prensa) ${ }^{6}$. Expondré brevemente algunas ideas bajtinianas a propósito de la naturaleza dialógica de la emisión, las cuales nos permitirán ver la complementariedad que existe entre sus respectivos puntos de vista, además de ofrecer un enfoque de análisis capaz de responder a la complejidad del diálogo.

Con objeto de situar el estudio del lenguaje bajo una óptica superadora de las contradicciones en que se hallaba inmerso a comienzos de siglo (subjetivismo idealista vs. objetivismo abstracto), Bajtin vio en el concepto de emisión una de las claves para desarrollar su peculiar perspectiva metodológica. Desde su punto de vista, la emisión debe ser analizada como un instrumento al servicio de la comunicación, capaz de organizar la experiencia humana (Volosinov y Bajtin: Marxism and Philosophy of Language, 1986, p. 85). Con la óptica que la distancia histórica nos aporta, podemos incluir este concepto entre los procedimientos semióticos de mediación de la actividad, incorporándolo a una terminología y a un enfoque que entronca con la mejor tradición vygotskiana (Wertsch, 1985; en prensa). Así pues, las razones para considerarla como instrumento analítico y puntualizar su valor de mediador semiótico en el curso de las acciones humanas estriba, entre otras cosas, en la doble dirección en que se orienta la emisión: la comunicación y la experiencia individual.

Después de lo que se ha apuntado, la perspectiva propuesta por Bajtin es dialógica en su acepción más estricta, pues la interacción social en la que todo enunciado encuentra su sentido, se desarrolla única y exclusivamente a través del diálogo (idem., p. 95). Como Bajtin dice al respecto, «cualquier emisión, ..., es sólo un momento del proceso continuo de la comunicación verbal", de la interacción social (aquí y abora); por esa razón, "la comunicación verbal no puede ser comprendida ni explicada fuera de sus conexiones con una situación concreta” (ibid., p. 95). A mi juicio, la propues- 
ta del autor es bien clara: el análisis de cada enunciado debe hacerse tomando en consideración todos los demás y, muy especialmente, los que le anteceden en el plano del diálogo. Por otra parte, dado que la noción bajtiniana de comunicación se sitúa en unas dimensiones conductuales muy puntuales, la situación concreta en que se desarrolla el diálogo es otro factor clave que no debe desdeñarse.

En cuanto al tema que aquí nos ocupa (la base dialogal desde la que emerge el $\mathrm{HE}$ ), las ideas de Bajtin tienen al respecto una vigencia mayor de lo que en un primer momento podría pensarse. La aplicación de estas ideas pueden plasmarse en la siguiente formulación: el análisis de cualquier suceso de habla en la producción verbal del niño obliga a considerar por igual dos tipos de contexto: el contexto lingüistico (todo lo que los hablantes han dicho hasta ese momento preciso) y el contexto extralingüistico (i. e., los objetos sobre los que se actúa y de los que se habla). Este último representa un aspecto muy importante de la situación concreta a que alude Bajtin, siendo en gran medida responsable de una buena parte de las producciones verbales infantiles. Así pues, la perspectiva dialógica nos permite atender por igual a la interdependencia entre emisiones (plano conversacional), y a la relación entre éstas y los objetos y sucesos físicos en que se desarrollan las acciones del niño (contexto extralingüístico). Esta doble proyección de la noción bajtiniana de emisión, nos pone sobre la pista de una forma nueva de abordar el estudio del habla egocéntrica. Si toda emisión se debe estudiar en el marco del diálogo y éste sólo puede existir por la acción conjugada de dos o más participantes (hablante-oyente, hablante-audiencia, etc.), las emisiones egocéntricas en particular tienen que recibir algún tipo de influjo de aquéllos que rodean al niño. Los próximos párrafos nos darán una imagen más exacta de lo que la perspectiva dialógica promete al respecto.

Los trabajos de Wertsch (Wertsch, 1979, 1980, en prensa; Wertsch y Stone, 1985), que comenzaron bajo la influencia directa de Vygotski y que en los últimos años han ido impregnándose de una fuerte inspiración bajtiniana, son un fiel reflejo del papel que cumple el diálogo en el desarrollo de la autorregulación. Como ya comenté brevemente cuando me referí al cuarto requisito conversacional (el otro como colaborador potencial), en la colaboración entre el adulto y el niño en la resolución de un problema (i. e., puzzle) es posible observar la transición entre las distintas formas de regulación de la acción a lo largo de las fases por las que transcurre dicha colaboración. Durante los primeros momentos de la misma, el adulto asumía una gran parte del control sobre la tarea (beterorregulación), mientras que en los últimos era el propio niño el que adquiría progresivamente una mayor responsabilidad sobre sus actos. La conexión genética entre las distintas fases resolutorias fue posible en la medida en que Wertsch y sus cols. consideraron la totalidad de la actividad y, consecuentemente, del diálogo. Se comprobaba así que las emisiones autorreguladoras (HE) no surgen en el vacío, sino que están en estrecha relación con las heterorreguladoras proferidas por el adulto al comienzo de la tarea. El descubrimiento de la relación genética existente entre ambas y, consecuentemente, entre el diálogo y el habla interna fue posible en la medida en que implícita o explícitamente se cumplía el postulado de Bajtin, según el cual, cada emisión representa un momento en la totalidad de la comunicación y, por tanto, debe ser con- 
siderada en el contexto de lo que ya se ha dicho. Las emisiones heterroreguladoras del adulto hicieron posible las posteriores autorreguladoras del niño. El nexo conductor que las une habría pasado desapercibido para un investigador que abordara monológicamente el análisis de cada una de ellas.

\section{Un ejemplo de dependencia conversacional entre producciones egocéntricas}

Desde mi punto de vista, el diálogo es un tema de interés no sólo para el estudio de la interacción adulto-niño. El enfoque dialógico debe ir más allá de las interacciones asimétricas y aplicarse también al estudio de las relaciones entre iguales. Las razones por las que se defiende en este artículo el diálogo como una cuestión fundamental a considerar tiene que ver con las formas de transición entre el habla social y el habla interna que las diferentes emisiones de $\mathrm{HE}$ revisten. El tratamiento que se da al episodio que expongo a continuación, extraído de una de las diadas perteneciente a la investigación ya comentada (Ramírez, en prensa), es un fiel reflejo del uso de esta perspectiva.

Dos niñas (L e I) están dibujando a sus familias respectivas. Una de ellas, L, cree haber terminado su dibujo y, sin mirar ni dirigirse explicitamente a su compañera exclama:

1. L. - «Ya, ya acabe, pues».

Más tarde, se fija detenidamente en el papel sobre el que dibuja y hace un gesto de sorpresa, como descubriendo que algo queda aún por hacer. A continuación, I, que ha conversado largamente con L sobre su "hermanito" y parece estar muy preocupada por dibujarlo bien, exclama:

2. I. - «Hermanito te dije, te dijo el señor».

3. I. - "Yo voy a hacer a mi papá...» ${ }^{\dagger}$.

4. L. - «mi mamá... Voy a pintarle el cabello, también los ojos, también la bocam.

5. I. - «Yo voy a hacer la mía. Yo voy a hacer todo».

(Las emisiones 3.I, 4.L y 5.I fueron proferidas mientras las niñas dibujaban, sin que la mirada se apartara en ningún momento del papel.)

Poco después, I se fija en el micrófono con el que se están registrando sus voces y trata de iniciar una conversación con L sobre éste. Esta última, L, no la presta atención y continúa con su tarea.

El interés de este episodio estriba en la interdependencia conversacional existente entre las emisiones 3.I, 4.L y 5.I. A pesar de su orientación a la acción en curso, ninguna de ellas puede analizarse desde una perspectiva monológica exclusivamente. Las tres tienen un potencial valor regulador y su carácter privado está garantizado en gran medida por rasgos extraconversacionales (no existe contacto explícito entre los interlocutores), cognitivos (se orientan a los objetivos de la tarea) y atencionales (las niñas no dejan de dibujar mientras hablan). Sin embargo, hay dos aspectos dignos de consideración, que ponen de manifiesto la fuerte dependencia dialogal entre las tres emisiones contenidas en este episodio: 1) un ritmo de producción con características similares al turno de palabras (turn-talking); 2) una marcada dependencia sintáctica entre las oraciones que estos enunciados incluyen. En ellas como en otras emisiones y episodios del estudio referido, los solapamientos son casi inexistentes. Un orden de producción 
muy parecido al turno de palabras, típico de toda conversación, se hace presente a lo largo de la práctica totalidad de las interacciones registradas. Cuando los niños hablan egocéntricamente no lo hacen a la vez sino que cada emisión se realiza inmediatamente después de que ha finalizado la de su compañero. En cuanto al segundo apartado, la dependencia sintáctica en que se encuentran 4.L y 5.I está fuera de toda duda. Si hipotéticamente consideramos que el hecho de tomar un enunciado como propio sin considerar a nuestro interlocutor como su verdadero artífice es fiel reflejo de una actitud egocéntrica, entonces 4.L (a mi mamá...) representa un perfecto ejemplo de esta actitud. En ella, la hablante asume la emisión anterior (3.I -yo voy a hacer a mi papá-) sin tomar en consideración que es I la verdadera autora de la misma. Sujeto (yo) y verbo (voy) quedan excluidos de 4.L, aprovechándose integramente la sintaxis de lo que se ha dicho hasta ese momento. En el caso de 5. .I (yo voy a hacer la mía...) volvemos a observar una estrecha dependencia sintáctica, si bien la actitud egocéntrica a la que en términos hipotéticos me he referido, no parece reflejarse en igual proporción. En este último caso, la hablante (I) vuelve también a usar para sí la oración anterior, aunque esta vez con la adecuada pronominalización del elemento que se repite en ambas oraciones (mamá - la mía).

En las emisiones comentadas, como en otras muchas emisiones de carácter egocéntrico, se cumplen los requisitos dialógicos que Bajtin propone: ninguna de ellas puede ser considerada aisladamente y todas se encuentran unidas por el fino hilo del diálogo; a su vez, están estrechamente relacionadas con la situación inmediata, que, para el caso que nos ocupa, es tanto como decir el contexto extralingüístico en que las hablantes se encuentran inmersas. En este último se sitúan los objetivos que rigen su actividad y, consiguientemente, los temas que dominan el curso de la conversación.

\section{CONCLUSIONES}

Las hipótesis de Vygotski sobre el desarrollo de la autorregulación se articula en torno su noción de habla egocéntrica. Su característica más peculiar es la falta de diferenciación entre el habla para los demás (habla social) y el habla para uno mismo (habla interna).

La indiferenciación funcional de toda producción egocéntrica invita a desarrollar una metodología que, parafraseando a Wertsch, contemple por igual su origen (social), su sentido (orientado a la regulación de la tarea) y su destino (la posterior internalización).

Para la observación y el estudio del HE es necesario respetar una serie de requisitos conversacionales que, además de ser fundamentales en cuanto a la producción de semejante tipo de emisiones, evidencian su origen social.

Uno de los requisitos conversacionales a considerar, al que no se le ha prestado aún la suficiente atención, es el contexto extralingüístico en que se desenvuelve la tarea. La dificultad para compartir un contexto común reduce por igual el diálogo entre los niños y su más directo asociado, el $\mathrm{HE}$.

Si Vygotski exṕlicitó el origen social de esta forma de habla, Bajtin nos ofrece la posibilidad de estudiarlo desde una perspectiva dialógica, la cual 
da respuesta a la dependencia conversacional que parece observarse entre algunas de las emisiones incluidas en ella.

La perspectiva dialógica nos permite profundizar en el carácter indiferenciado de que hace gala esta forma intermedia entre el hablar para los demás y el hablar para uno mismo. La relación conversacional que manifiestan muchas de las emisiones egocéntricas sólo puede ser estudiada a través de semejante propuesta metodológica.

\section{Notas}

${ }^{1}$ El concepto de emisión que utilizado a lo largo de todo este artículo es sinónimo de expresión y tendria en utterance su equivalente inglés más próximo. Se incluiría en esta noción tanto el significado de la palabra o la oración como los rasgos prosódicos y entonativos que la acompañan.

${ }^{2}$ El término mediacionismo se emplea aquí para hacer alusión al conductismo mediacionista que tanto auge tuvo durante la década de los cincuenta y que representaba una versión muy diferente del concepto de mediación semiótica empleado por Vygotski. Para una mayor profundización en los planteamientos semióticos de esta escuela se puede consultar el libro de Ch. Osgood, G. J. Suci y P. H. Tannenbaum (1957): The Measurement of Meaning. University of Illinois Press (ed. española de J. Seoane y J. Bernia. La medida del significado. Madrid: Editorial Gredos, 1976).

${ }^{3}$ Con estas instrucciones se pretendian mantener las mismas condiciones que Frauenglass y Diaz recomiendan, cuando se desea observar una actuación verbal desinhibida por parte de los niños estudiados (Frauenglass y Díaz, 1985; trad. española: 1987).

${ }^{4}$ El término emisión sigue siendo válido para referirse a aquéllo que el niño «dice» en cada momento de la acción en curso. Sin embargo, es necesario precisar que para Vygotski, la unidad de análisis por excelencia para el estudio de la relación entre el pensamiento y el lenguaje era la palabra. Bajtin, por el contrario, centró su análisis sobre la emisión. Los recientes estudios en el marco del desarrollo del lenguaje y las investigaciones semióticas contemporáneas parecen conferir un mayor peso a la noción bajtiniana.

5 El concepto de acción que aquí se utiliza es el propuesto por Leontiev y la escuela soviética (Leontiev, 1973, 1981). La acción es un hecho molar, que en el caso que nos ocupa, combinan por igual la emisión verbal (funcionalmente reguladora) y el conjunto de operaciones que componen su parte motora.

- A pesar de vivir el mismo tiempo histórico, de recibir las mismas influencias culturales y de la similitud de puntos de vista, no existen datos que indiquen que ambos autores llegaran a conocerse.

${ }_{7}$ Las palabras escritas en cursiva indican una elevación del tono de voz y una cadencia especial en la forma en que ésta se articula.

\section{Referencias}

BERK, L. E. y GARVIN, R. A. (1984). Development of private speech among low-income Appalachian children. Developmental Psychology, 20, 271-286.

DíaZ, R. M. y PADILlA, K. A. (1985). Teoría e investigación empíricas sobre el lenguaje privado. Anuario de Psicología, 33 (2), 43-58.

Flavell, J. H. (1966). Le langage privé. Bulletin de Psychologie, 19, 698-701.

Frauenglass, M. H. y DiAz, R. M. (1985). Self-regulatory functions of children's private speech: a critical analysis of recent challenges to Vygotski's theory. Developmental Psycho$\log y, 21(2), 357-364$.

FusON, K. C. (1979). The development of self-regulating aspects of speech: A review. En G. Zivin (ed.). The development of self-regulation through private speech. Nueva York: John Wiley, 134-217.

GoudenA, P.P. (1983). Private speech: an analysis of its social and self-regulative funcions. Diss.: University of Utrecht.

GOUDENA, P. P. (1987). The social nature of private speech of preschoolers during problem solving. International Journal of Behavioral Development, 10 (2), 187-206.

Habermas, J. (1987). Teoria de la acción comunicativa. Vol. I. Racionalidad de la acción y racionalización social. Madrid: Taurus. 
HickmanN, M. (1980). Creating referentes in the discourse: A developmental analysis of linguistic cohesion. En J. Kreiman y A. Ojeda (eds.). Papers of parasession on pronouns and anaphora: Sixtheenth Regional Meeting of the Chicago Linguistic Society. Chicago: Chicago Linguistic Society.

HICKMANN, M. (1980). The context dependence of linguistic and cognitive proceses. En M. Hickmann (ed.). Proceeding of a Working Conference on the Social Fundations of Language and Thought. Chicago: Center of Psychosocial Studies.

HICKMANN, M. (1985). The implications of discourse skills in Vygotskian developmental theory. En J. V. Wertsch (ed.). Culture, communication, and cognition. Vygotskian perspectives. Nueva York: Cambridge University Press.

HoLQUIST, M. (1981). The politics of the representation: Selected Papers from the English Institute. Baltimore: Johns Hopkins University Press.

KARMILOFF-SMITH, A. (1979). A functional approach to child language. A study of determiners and reference. Nueva York: Cambridge University Press.

KOHLBERG, L., YAEGER, J. y HJERTHOLM, E. (1968). Private speech: Four studies and review of theories. Child Development, 39, 817-826.

Leontiev, A. N. (1973). The problem of activity in Psychology. Soviet Psychology. Winter, 4-33.

Leontiev, A. N. (1981). Problems of the development of the mind. Moscú: Progress Publishers.

PellegrinI, A. D. (1980). A pragmatic-semantic analysis of preschoolers' self-regulating speech. Communication and Cognition, 13 (4), 347-361.

PellegrinI, A. D. y DesTeFANO, J. S. (1979). The functions of private speech in preschool children. International Journal of Pshycholinguistics, 15, 27-42.

PIAGET, J. (1973). Le langage et la pensée chez l'enfant. Neuchâtel: Delachaux et Niestlé.

ReEse, H.W. (1962). Verbal mediation as a function of age level. Psychological Bulletin, 59, 502-509.

VolosinOV, V. N./Bajtin, M. M. (1986). Marxism and the philosophy of langage. Cambridge, Mass.: Harvard University Press.

VyGotski, L. S. (1973). Pensamiento y lenguaje. Buenos Aires: La Pléyade.

VYGOTSKI, L. S. (1979). El desarrollo de los procesos psicológicos superiores. Barcelona: Grijalbo.

WERTSCH, J. V. (1979). From social interaction to higher pshycological processes: A classification and application of Vygotsky's theory. Human Development, 2, 3-22.

WERTSCH, J. V. (1980). The significance of dialogue in Vygotsky's account of social, egocentric and inner speech. Contenporary Educational Psychology, 5, 150-162.

WerTSCH, J. V. (1985). The semiotic mediation of mental life: L. S. Vygotski and M. M. Bajtin. En E. Mertz, R. J. Parmentier (eds.). Semiotic mediation. Orlando, Florida: Academic Press, 49-71.

WerTSCH, J. V. (1985). Vygotski. The social formation of mind. Nueva York: Harvard University Press.

WERTSCH, J. V. (en preparación). Voices of the mind (Draft). Worcester, Mass.: Clark University.

WERTSCH, J. V. y STONE, C. A. (1985). The concept of internalization in Vygotsky's account of the genesis of higher mental functions. En J. V. Wertsch (ed.). Culture, communication and cognition. Vygotskian perspectives. Nueva York: Cambridge University Press.

\section{Extended Summary}

During the last two decades western psychologists have shown the importance of Vygotskian hypotheses in explaining the development of selfregulation skills. They paid attention to the role of these hypotheses in order to explain the relationships between thinking and speech. Studies on the development of egocentric speech allow us to understand its role in the genesis of regulation of actions, and they are useful in explaining social sources of mental life (Kohlberg, Yaeger \& Hjermholm, 1982; Frauenglass \& Díaz, 1985; Díaz, 1985; Fuson, 1979; Wertsch, 1979; Berk, 1984): The study of the genesis of regulation of actions was extended by James Wertsch and his colleagues through the analysis of problem solving tasks and adult- 
child interactions (Wertsch, 1979, 1980; Wertsch \& Hickman, 1987; Wertsch \& Stone, 1985; Wertsch, Minick \& Arns, 1984).

The Vygotskian hypotheses and related studies stimulated the author of this paper to propose a new methodology of analysis for future investigations on egocentric speech. The basis for this methodology may be found in the set of conversational requirements which have to be considered when the production of egocentric speech is studied. These requirements may be found in Vygotsky's research work and in several investigations by Western psychologists (Kohlberg, Yaeger \& Hjermholm, 1982; Wertsch, 1979; Frauenglass \& Díaz, 1985; Goudena, 1987; Ramírez, in press).

Another important aspect of egocentric speech which has not received too much attention from European and American researchers is its undifferentiated nature. The differentiation between speech directed to others and to oneself was one of the central points in Vygotskian perspective, its analysis allow us to understand the origin of private events. To extend the Vygotskian hypothesis, attention must be, therefore, focused on the dialogical dimension of private speech, which was developed by Bakhtin. This author, as Wertsch has pointed, shared with Vygotsky common theoretical viewpoints.

Here two different episodes of conversation were analyzed in order to highlight the importance of Bakhtin's proposals. In both of them, two characteristics that were observed to occur not only in egocentric utterances but also in social utterances were analyzed; these were: a) Egocentric utterances have a very similar sequence to the one which may be observed in turn taking in everyday conversation, b) their meanings are clearly of dialogical interdependence. According to these observations, we propose a shift from monological analysis, focusing on isolated utterances, to dialogical analysis in which the meaning of each utterance is considered within the context in which they were performed. In other words, in the linguistic and communicative context of previous utterances. 